ORIGINAL ARTICLE

\title{
Biometric relationships between body size and otolith size in 15 demersal marine fish species from the northern Brazilian coast
}

\author{
Rory Romero de Sena OLIVEIRA ${ }^{1 *} \oplus$, Marcelo Costa ANDRADE ${ }^{1}$, Fabiola Seabra MACHADO르, \\ Élida Jesana Santana CUNHA ${ }^{1}$, Flaviane Souto de FREITAS ${ }^{1}$, Alex Garcia Cavalleiro de Macedo KLAUTAU², \\ Tommaso GIARRIZZOํㅜ, Ulrich SAINT-PAUL ${ }^{3}$ \\ Universidade Federal do Pará, Grupo de Ecologia Aquática, Núcleo de Ecologia Aquática e Pesca da Amazônia, Belém, Pará, Brazi \\ Instituto Chico Mendes de Conservação da Biodiversidade, Centro de Pesquisa e Gestão de Recursos Pesqueiros do Litoral Norte, Belém, Pará, Brazil \\ Leibniz-Zentrum für Marine Tropenforschung (ZMT), Bremen, Germany \\ Corresponding author: rorysena@ymail.com; $\mathbb{D}$ https://orcid.org/0000-0002-1411-9059
}

\begin{abstract}
The relationships between fish size and sagitta otolith measurements were calculated for the first time for 15 species belonging to six families from the northern Brazilian coast. A total of 220 fish were sampled from the bycatch landed by the bottom-trawl industrial shrimp-fishing fleet between August and September 2016. All species had strong relationships between otolith measurements and fish total length with the coefficient of determination $\left(r^{2}\right)$ ranging between 0.71 and 0.99 . The variable most strongly related to fish total length was found to be the sagittal otolith length (OL) with $98 \%$ of the variability. These relationships are a useful tool to estimate length and mass of preyed fish from otoliths found in stomach contents of marine predators.
\end{abstract}

KEYWORDS: Amazon delta, bycatch, sagitta, Neotropics, Sciaenidae, stomach content

\section{Relação biométrica entre tamanho coporal e tamanho do otólito em 15 espécies de peixes marinhos demersais da costa norte do Brasil}

\section{RESUMO}

As relaçóes entre o tamanho corporal do peixe e as medidas do otólito sagitta foram calculadas pela primeira vez para 15 espécies pertencentes a seis famílias de peixes demersais da costa norte do Brasil. Um total de 220 peixes foi amostrado da captura acidental realizada pelo arrasto de fundo da frota pesqueira industrial de camaráo entre agosto e setembro de 2016. Todas as espécies apresentaram fortes relaçóes entre as medidas do otólito e o comprimento total do peixe, com o coeficiente de determinação $\left(r^{2}\right)$ variando entre 0,71 e 0,99 . A variável mais fortemente relacionada ao comprimento total dos peixes foi o comprimento do otólito sagital (OL) com $98 \%$ da variabilidade. Essas relaçóes representam uma ferramenta útil para estimar o comprimento e a massa de peixes predados a partir dos otólitos encontrados no conteúdo estomacal de predadores marinhos.

PALAVRAS-CHAVE: Delta do Amazonas, captura acidental, sagitta, Neotrópico, Scianidae, conteúdo estomacal

\section{INTRODUCTION}

Otoliths are structures composed mainly of calcium carbonate, located in the inner ear of Osteichthyes, which have body balancing and hearing as main functions (Campana 2004). These structures are arranged in pairs, called sagitta, asteriscus, and lapillus in bony fish, and vary widely in size and shape among species (Campana 2004; Popper et al. 2005). Due to its largest size in the majority of bone fishes, sagittae otoliths are the most suitable for systematic and ecological studies, for taxon identification, age estimation, and life history tracking (Harvey et al. 2000;
De La Cruz-Agüero et al. 2016; Assis et al. 2018). Their nondigestible calcified structure has allowed them to be widely used to identify fishes ingested by different predators such as aquatic mammals, seabirds and fishes (Battaglia et al. 2010; Tuset et al. 2010). Furthermore, relationships between fish size and otolith measures are useful to elucidate the feeding behavior of piscivorous fauna, providing subsidies for the management of these species (De Pierrepont et al. 2005; Lombarte et al. 2006; Battaglia et al. 2010).

Studies on otoliths in marine fish from the Western South Atlantic are scarce (Waessle et al. 2003; Assis et al. 
2018; Souza et al. 2019). The high nutrient and sediment load from the Amazon River, near the Northern Coast of Brazil favors the occurrence of abundant fishery stocks, mainly shrimp (Penaeidae) and several fish species (Isaac and Braga 1999; Marceniuk et al. 2019). The ichthyofauna is functionally important as an intermediate trophic level for many consumers, however, its importance is not well understood (Barletta et al. 2010).

The present study aimed to determine the relationship between fish size (i.e. length and weight) and sagittae otolith measurements (i.e. length, width, and weight) for the 15 most abundant demersal species captured along the northern Brazilian coast.

\section{MATERIAL AND METHODS}

Fish were captured between August and September 2016 in an area characterized by the estuarization of inshore waters, due to the proximity to the Amazonas River $\left(5^{\circ} 02^{\prime} 21.6^{\prime \prime} \mathrm{N}\right.$, 4749'33.9”W; 0 51'02.0” N, 4750'30.0”W northern and southern limits of the sampling area, respectively; Figure 1), and is inserted in the world's second largest mangrove $(-700$ $000 \mathrm{ha}$ ), which is an important fishery area (Isaac and Braga 1999; Giarrizzo and Krumne 2008).

Samples were randomly collected from the bottom trawl of industrial shrimp trawlers using a $30 \times 21 \mathrm{~mm}$ mesh bottom trawl of $22.4 \mathrm{~m}$ length towed at velocity of -2.5 knots at $35-49 \mathrm{~m}$ depth. Bycatch were taken to the laboratory and kept frozen until processing. Sampled fish were identified to species level, and measured (standard

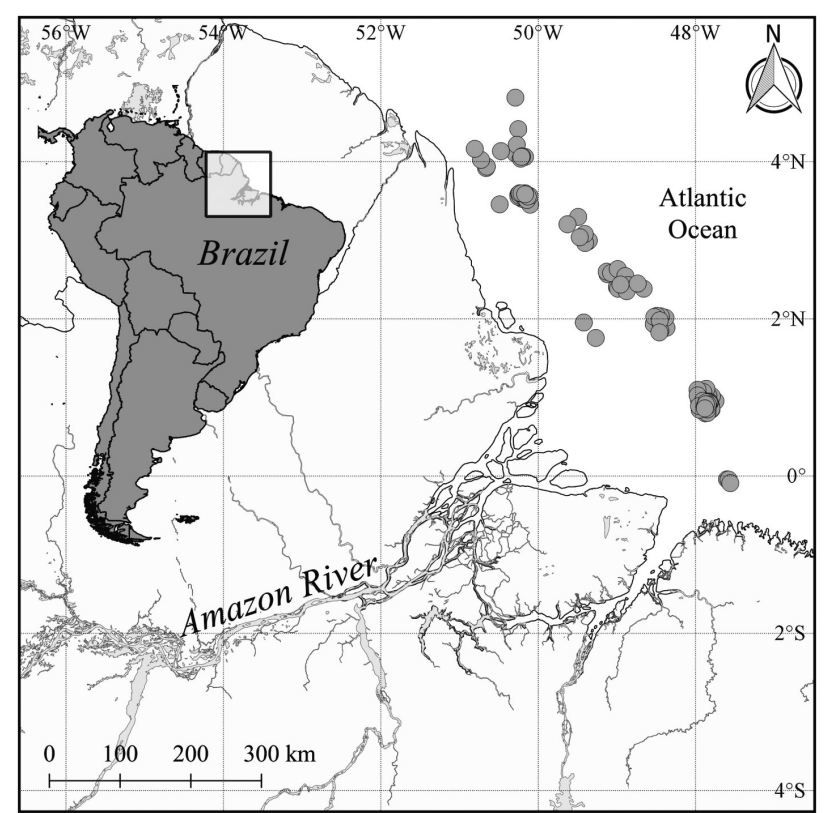

Figure 1. Sampling locations of the study on the Northern Brazilian Coast. Samples of demersal fish were collected from bottom trawling of industrial shrimp trawlers along the coast of Pará and Amapá states in Brazil (grey dots). length, SL, and total length, TL, precision $0.01 \mathrm{~cm}$ ) and weighed for total body weight (BW, $0.01 \mathrm{~g}$ ). Sagittae otoliths were removed, cleaned and stored dry in coded microtubes. Each otolith was weighed (WO, $0.0001 \mathrm{~g}$ ) using an analytical balance, and measured for maximum length (OL, 0.001 $\mathrm{cm})$, as the horizontal distance between the anterior and posterior tips of the sagitta, and width $(\mathrm{OW}, 0.001 \mathrm{~cm})$, as the greater distance between the dorsal and ventral margins of the otolith (Harvey et al. 2000; Battaglia et al. 2010).

Vouchers of each species were fixed in $10 \%$ formalin after processing, then preserved in 70\% alcohol and deposited in the ichthyological collection of the Grupo de Ecologia Aquática (GEA) at Universidade Federal do Pará (UFPA). Potential differences between the dimensions of the right and left sagittae otoliths were tested using a paired Student's t-test per species (Park et al. 2018). The length-length relationship (LLR) was determined by the method of least squares to fit a simple linear regression model: $\mathrm{TL}=a+b S \mathrm{~L}$. The length-weight relationship (LWR) was determined as: W $=a S L^{b}$, and was fitted to the data using a linear regression of the $\log _{10}$-transformed data. Morphometric relationships between TL and otolith dimensions were calculated using linear $(\mathrm{Y}=a+b \mathrm{X})$ and linearizable $\left(\mathrm{Y}=a \mathrm{X}^{b}\right)$ regression models, which best fit the data. When present, outliers were removed by graphical inspection of the plot before performing the regression analyses (Froese et al. 2011). The coefficient of determination (Pearson $r$-squared, $r^{2}$ ) was used as indicator of regression quality and to check if fish growth (b) was statistically different from isometric growth, a t-test $\left(H_{0}: b=3\right)$ (Froese et al. 2011). A significance level of $\alpha<$ 0.05 (confidence level $\pm 95 \%$ ) was routinely adopted.

\section{RESULTS}

The analyses were performed using 220 specimens from 15 species (see Figure 2 for otoliths) and six families. The most representative family was Sciaenidae with nine species, followed by Haemulidae, with two species, and the remaining four families, with only one species each (Table 1). Body weight ranged between 6.7 to $911.1 \mathrm{~g}$, SL between 7.1 to $69.5 \mathrm{~cm}$, and TL from 8.9 to $72.1 \mathrm{~cm}$. Paired t-tests did not detect differences between left and right sagittae otoliths for OL, OW, and WO ( $\mathrm{p}>0.05$ for all species). Hence, all further analyses were standardized by using only the left otolith measures.

Differences between the $b$ values estimated by the different regression models were species specific. However, for species of the same family with similar body shape (e.g. Sciaenidae and Haemulidae), values were similar (Figure 3). Length-weight relationships (LWR) were highly significant $(\mathrm{p}<0.001)$ only for six species: Ctenosciaena gracilicirrhus, Macrodon ancylodon, Menticirrhus americanus (all Sciaenidae), Haemulon steindachneri (Haemulidae), 


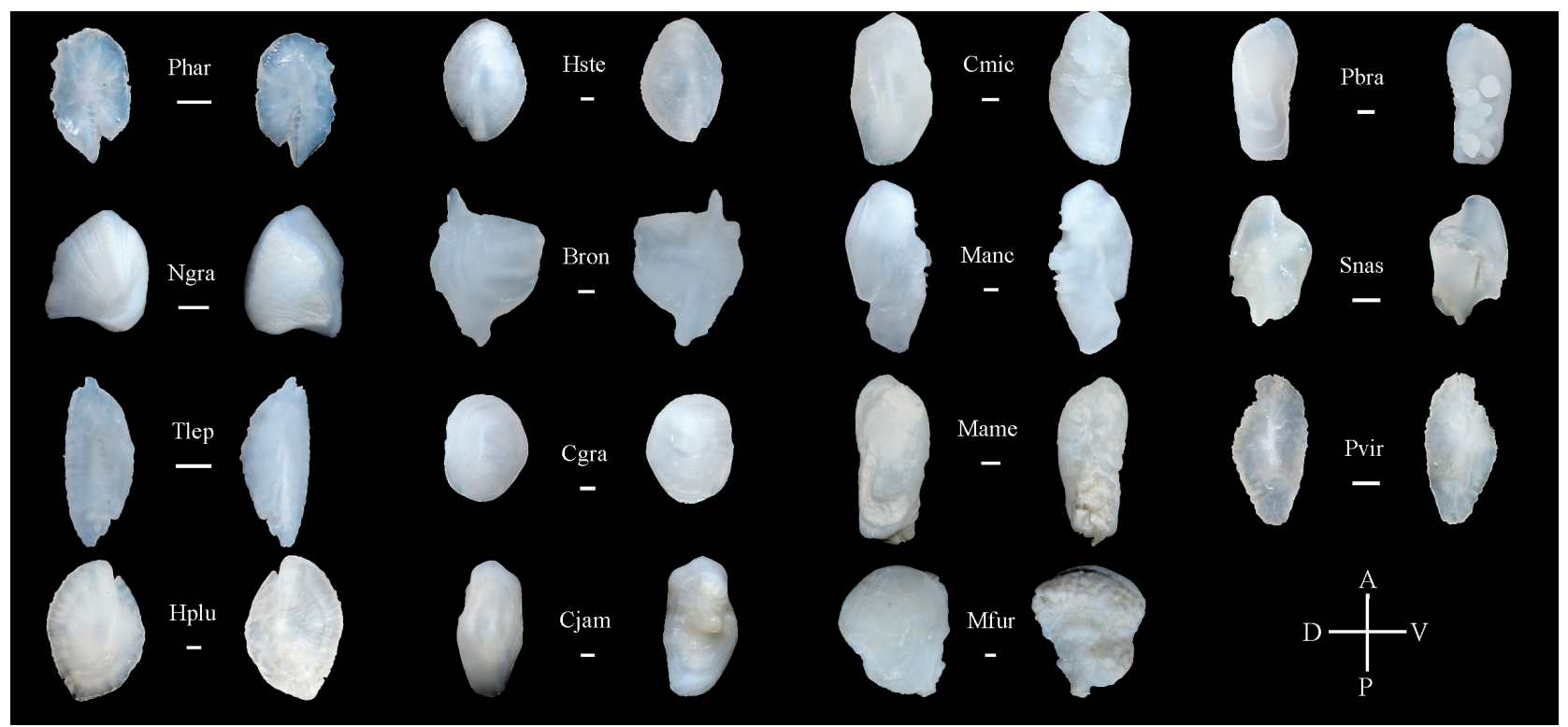

Figure 2. Left sagitta otolith morphology of the 15 fish species captured by bottom trawling along the northern Brazilian coast. Phar: Pellona harroweri, Ngra: Notarius grandicassis, Tlep: Trichiurus lepturus, Hplu: Haemulon plumierii, Hste: Haemulon steindachneri, Bron: Bairdiella ronchus, Cgra: Ctenosciaena gracilicirrhus, Cjam: Cynoscion jamaicensis, Cmic: Cynoscion microlepidotus, Manc: Macrodon ancylodon, Mame: Menticirrhus americanus, Mfur: Micropogonias furnieri, Pbra: Paralonchurus brasiliensis, Snas: Stellifer naso, Pvir: Polydactylus virginicus, D: dorsal, V: ventral, A: anterior, P: posterior. Scale bars $=1 \mathrm{~mm}$. This figure is in color in the electronic version.

Table 1. Sample size (N) and ranges of the standard length (SL), total length ( $T L$ ), weight (W), otolith length (OL) and otolith width (OW) of 15 demersal fish species captured by bottom trawl in the Northern Brazilian coast in 2016.

\begin{tabular}{|c|c|c|c|c|c|c|c|c|}
\hline Family/Species & Voucher & N & $\mathrm{SL}(\mathrm{cm})$ & $\mathrm{TL}(\mathrm{cm})$ & $W(g)$ & $\mathrm{OL}(\mathrm{cm})$ & OW (cm) & WO (g) \\
\hline \multicolumn{9}{|l|}{ Pristigasteridae } \\
\hline Pellona harroweri (Fowler, 1917) & GEA4157 & 9 & $10.5-13.2$ & 13.8-14.6 & $23.98-28.35$ & $0.131-0.445$ & $0.079-0.271$ & $0.0068-0.0099$ \\
\hline \multicolumn{9}{|l|}{ Ariidae } \\
\hline Notarius grandicassis (Valenciennes, 1840) & GEA4154 & 10 & $14.7-42.3$ & $17.5-46.5$ & 50.38-911.12 & $0.111-0.233$ & $0.088-0.193$ & $0.1242-1.2638$ \\
\hline \multicolumn{9}{|l|}{ Trichiuridae } \\
\hline Trichiurus lepturus Linnaeus, 1758 & & 10 & $43.3-69-5$ & $47.7-72.1$ & 63.33-232.92 & $0.371-0.540$ & $0.156-0.204$ & $0.0035-0.0103$ \\
\hline \multicolumn{9}{|l|}{ Haemulidae } \\
\hline Haemulon plumierii (Lacepède, 1801) & GEA4164 & 14 & $14.5-19.0$ & $17.5-20.9$ & $94.75-159.98$ & $0.097-0.170$ & $0.080-0.125$ & $0.0619-0.1715$ \\
\hline Haemulon steindachneri (Jordan \& Gilbert, 1882) & GEA4662 & 13 & $14.2-18.2$ & 19.3-22.1 & 105.3-168.03 & $0.141-0.196$ & $0.095-0.120$ & $0.1041-0.1223$ \\
\hline \multicolumn{9}{|l|}{ Sciaenidae } \\
\hline Bairdiella ronchus (Cuvier, 1830) & & 8 & $19.2-31.4$ & 23.4-37.6 & 207.76-756.00 & $0.145-0.254$ & $0.095-0.210$ & $0.0124-2.6202$ \\
\hline Ctenosciaena gracilicirrhus (Metzelaar, 1919) & GEA4166 & 26 & 7.1-13.4 & 8.9-16.4 & $9.29-73.74$ & $0.446-0.735$ & $0.380-0.582$ & $0.0242-0.1091$ \\
\hline Cynoscion jamaicensis (Vaillant \& Bocourt, 1883) & & 20 & $12.3-21.5$ & $16.3-23.0$ & $47.91-160.14$ & $0.880-1.221$ & $0.446-0.613$ & $0.0613-0.2203$ \\
\hline Cynoscion microlepidotus (Cuvier, 1830) & & 13 & $14.5-34.5$ & $17.0-38.5$ & $39.70-625.60$ & $0.139-0.260$ & $0.074-0.140$ & $0.0747-0.3090$ \\
\hline Macrodon ancylodon (Bloch \& Schneider, 1801) & GEA3702 & 31 & $14.4-25.3$ & $18.2-30.9$ & $47.20-242.20$ & $0.943-1.341$ & $0.431-0.709$ & $0.0618-0.1979$ \\
\hline Menticirrhus americanus (Linnaeus, 1758) & GEA4311 & 16 & $9.5-14.0$ & $12.0-17.0$ & $22.73-82.02$ & $0.088-0.120$ & $0.075-0.092$ & $0.0468-0.1079$ \\
\hline Micropogonias furnieri (Desmarest, 1823) & GEA4299 & 14 & $12.1-27.8$ & $17.8-33.9$ & $51.32-448.70$ & $0.150-0.247$ & $0.060-0.187$ & $0.0518-0.7530$ \\
\hline Paralonchurus brasiliensis (Steindachner, 1875) & GEA4914 & 10 & $11.0-18.5$ & $15.6-22.0$ & $38.20-129.00$ & $0.140-0.193$ & $0.067-0.093$ & $0.0587-0.0913$ \\
\hline Stellifer naso (Jordan, 1889) & GEA4715 & 15 & $7.7-8.1$ & 9.4-11.2 & $6.70-13.12$ & $0.386-0.433$ & $0.243-0.296$ & $0.0118-0.0133$ \\
\hline \multicolumn{9}{|l|}{ Polynemidae } \\
\hline Polydactylus virginicus (Linnaeus, 1758) & GEA4834 & 11 & 11.9-19.0 & $17.0-25.3$ & $42.39-177.99$ & $0.415-0.574$ & $0.226-0.287$ & $0.0052-0.0128$ \\
\hline
\end{tabular}



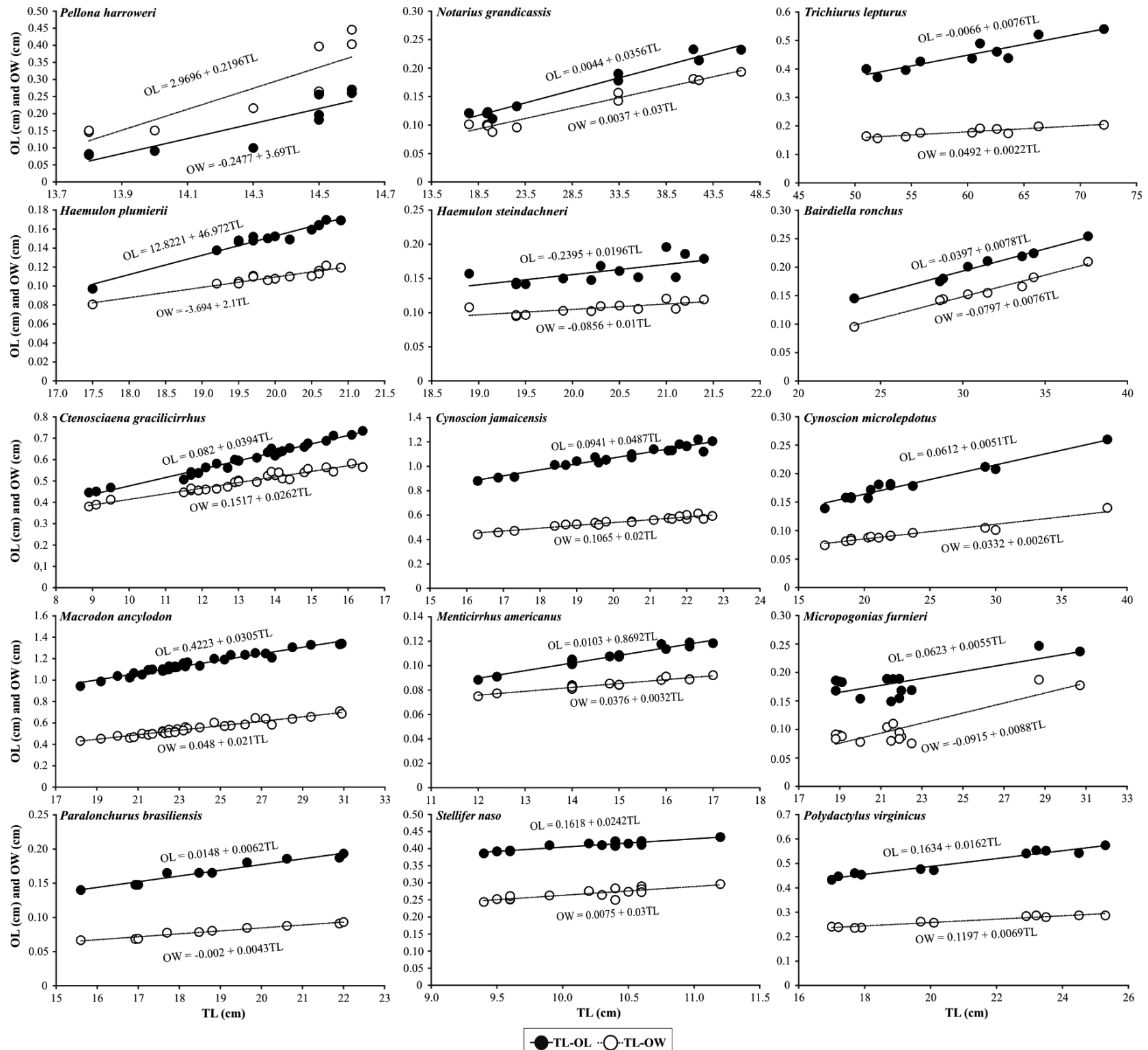

Figure 3. Relationship of otolith length (OL) and width (OW) with total length (TL) of 15 fish species captured by bottom trawling along the northern Brazilian coast.

Pellona harroweri (Pristigasteridae), and Polydactylus virginicus (Polynemidae) (Table 2). However, all $r^{2}$ values were greater than 0.91 in LWRs. The slope $b$ of the LWR ranged from 2.75 for Notarius grandicassis to 3.54 for $H$. steindachneri. Otolith-fish body biometric relationships (i.e. BW-OL, BW-WO, BW-OW, TL-OL, TL-OW, WO-TL, WO-OL, and WO-OW) were highly correlated with $r^{2}$, ranging from 0.713 in TL-OW for Stellifer naso to 0.980 in TL-OL for Bairdella ronchus, and most of the relationships were significant $(\mathrm{p}<0.01)$ (Table 2).

\section{DISCUSSION}

Body size and mass relationships are important tools for the functional understanding of a species at specific locations
(Froese et al. 2011). Generally the length-weight relationships (LWRs) are used for converting lengths into fish mass and vice versa (Froese 2006; Froese et al. 2011), and length-length relationships (LLRs) are used to convert one length into another (e.g. standard length to total length). Additionally, assuming that otolith size is closely correlated to fish size, and its shape is species specific (Campana 2004), it is suggested that otolith analysis is a feasible and reliable method to identify fish species and to estimate fish size and weight (Battaglia $e t$ al. 2010; Park et al. 2018). Froese (2006) suggests that the angular coefficient does not differ from isometry when $\mathrm{b}=$ 3. Accordingly our $b$ values of the BW-TL relationship were isometric for sciaenids, which, in addition, had coefficients of determination $\left(r^{2}\right)$ higher than 0.90 (see Figure 3). However, 
Table 2. Estimated parameters of the relationship between measurements of fish and sagitta otoliths in 15 demersal fish species captured by bottom trawl along the northern Brazilian coast in 2016.

\begin{tabular}{|c|c|c|c|c|c|c|c|c|c|c|c|c|c|c|}
\hline \multirow{2}{*}{ Species } & \multicolumn{7}{|c|}{ SL-TL } & \multicolumn{7}{|c|}{ BW-TL } \\
\hline & $\mathrm{a}$ & SE $a$ & $b$ & SE $b$ & $r^{2}$ & $G$ & Model & $\mathrm{a}$ & $\mathrm{SE} a$ & $b$ & SE $b$ & $r^{2}$ & G & Model \\
\hline Pellona harroweri & 0.2838 & 0.025 & 7.77 & 0.362 & 0.95 & + & L & 0.0104 & 1.0308 & 2.9513 & 0.0114 & 0.99 & - & $\mathrm{NL}$ \\
\hline Notarius grandicassis & 1.03 & 0.032 & 2.81 & 0.899 & 0.99 & I & L & 2.2292 & 1.0617 & 0.6588 & 0.0596 & 0.96 & - & $\mathrm{NL}$ \\
\hline Trichiurus lepturus & 0.7969 & 0.023 & 16 & 1.281 & 0.99 & + & L & 0.0002 & 3.5751 & 3.209 & 0.3127 & 0.93 & । & $\mathrm{NL}$ \\
\hline Haemulon plumierii & 0.6535 & 0.089 & 8.96 & 1.498 & 0.82 & + & L & 0.0176 & 1.4708 & 2.9973 & 0.129 & 0.98 & । & $\mathrm{NL}$ \\
\hline Haemulon steindachneri & 0.6209 & 0.022 & 10.1 & 0.365 & 0.99 & + & L & 0.0029 & 1.1984 & 3.5416 & 0.06 & 1 & + & $\mathrm{NL}$ \\
\hline Bairdiella ronchus & 1.142 & 0.144 & 1.28 & 3.778 & 0.91 & I & L & 0.0275 & 1.6226 & 2.8464 & 0.1412 & 0.99 & । & $\mathrm{NL}$ \\
\hline Ctenosciaena gracilicirrhus & 1.0738 & 0.044 & 1.77 & 0.475 & 0.96 & - & L & 0.0042 & 1.3198 & 3.4748 & 0.108 & 0.98 & + & $\mathrm{NL}$ \\
\hline Cynoscion jamaicensis & 0.8012 & 0.041 & 6.11 & 0.722 & 0.96 & + & L & 0.0055 & 1.8035 & 3.2789 & 0.1965 & 0.94 & । & NL \\
\hline Cynoscion microlepidotus & 1.0567 & 0.034 & 2.48 & 0.697 & 0.99 & । & L & 0.0043 & 2.4226 & 3.2398 & 0.2833 & 0.92 & । & $\mathrm{NL}$ \\
\hline Macrodon ancylodon & 1.0541 & 0.055 & 3.74 & 1.053 & 0.93 & I & L & 0.0026 & 1.5049 & 3.3368 & 0.1291 & 0.96 & + & $\mathrm{NL}$ \\
\hline Menticirrhus americanus & 1.107 & 0.043 & 1.39 & 0.518 & 0.98 & - & L & 0.0038 & 1.5617 & 3.5124 & 0.166 & 0.97 & + & $\mathrm{NL}$ \\
\hline Micropogonias furnieri & 0.7445 & 0.029 & 9.31 & 0.513 & 0.98 & + & L & 0.0041 & 2.5242 & 3.2587 & 0.3014 & 0.91 & । & $\mathrm{NL}$ \\
\hline Paralonchurus brasiliensis & 0.8536 & 0.093 & 6.68 & 1.351 & 0.91 & + & L & 0.0067 & 2.5334 & 3.1352 & 0.3168 & 0.92 & I & $\mathrm{NL}$ \\
\hline Stellifer naso & 0.2287 & 0.004 & 5.59 & 0.042 & 1 & + & L & 0.0038 & 1.8594 & 3.3745 & 0.2676 & 0.92 & । & $\mathrm{NL}$ \\
\hline \multirow[t]{3}{*}{ Polydactylus virginicus } & 1.2467 & 0.081 & 1.39 & 1.277 & 0.96 & । & L & 0.0028 & 1.3329 & 3.4219 & 0.0949 & 0.99 & + & $\mathrm{NL}$ \\
\hline & \multicolumn{7}{|c|}{ BW-OL } & \multicolumn{7}{|c|}{ BW-OW } \\
\hline & a & $\mathrm{SE} a$ & $b$ & SE $b$ & $r^{2}$ & $G$ & Model & a & SE $a$ & $b$ & SE $b$ & $r^{2}$ & G & Model \\
\hline Pellona harroweri & -7.1025 & 0.7331 & 9.462 & 1.0914 & 0.93 & + & $\mathrm{NL}$ & -0.1292 & 0.014 & 1.31 & $1 \mathrm{E}-04$ & 0.92 & - & $\mathrm{NL}$ \\
\hline Notarius grandicassis & 2228.98 & 2.664 & 4.9264 & 0.3438 & 0.89 & + & $\mathrm{NL}$ & 1571.8 & 2.748 & 5.11 & 0.396 & 0.87 & + & NL \\
\hline Trichiurus lepturus & 1523.7 & 1.4599 & 3.2232 & 0.4627 & 0.86 & I & $\mathrm{NL}$ & 0.0706 & 1.142 & 0.2 & 0.028 & 0.86 & - & $\mathrm{NL}$ \\
\hline Haemulon plumierii & 823.392 & 1.1803 & 0.942 & 0.087 & 0.91 & - & $\mathrm{NL}$ & 2700.3 & 1.274 & 1.34 & 0.109 & 0.93 & - & $\mathrm{NL}$ \\
\hline Haemulon steindachneri & 0.0491 & 0.0095 & 0.0009 & 0.0001 & 0.93 & - & L & 0.0207 & 1.276 & 0.35 & 0.05 & 0.81 & - & $\mathrm{NL}$ \\
\hline Bairdiella ronchus & 0.1077 & 0.0075 & & $1 \mathrm{E}-05$ & 0.97 & - & L & & & 0.55 & 0.046 & 0.96 & - & NL \\
\hline Ctenosciaena gracilicirrhus & 0.2616 & 1.0262 & 0.2388 & 0.0074 & 0.98 & - & $\mathrm{NL}$ & 0.2535 & 1.028 & 0.19 & 0.008 & 0.96 & - & $\mathrm{NL}$ \\
\hline Cynoscion jamaicensis & 0.3527 & 1.0978 & 0.24 & 0.0201 & 0.89 & - & $\mathrm{NL}$ & 0.1906 & 1.098 & 0.23 & 0.02 & 0.87 & - & $\mathrm{NL}$ \\
\hline Cynoscion microlepidotus & 0.0712 & 1.1087 & 0.1972 & 0.0219 & 0.88 & - & $\mathrm{NL}$ & 0.0398 & 1.123 & 0.18 & 0.025 & 0.83 & - & $\mathrm{NL}$ \\
\hline Macrodon ancylodon & 0.4867 & 1.0457 & 0.1866 & 0.0097 & 0.93 & - & $\mathrm{NL}$ & & 1.089 & 0.26 & 0.018 & 0.87 & - & $\mathrm{NL}$ \\
\hline Menticirrhus americanus & 0.042 & 1.0605 & 0.2412 & 0.0152 & 0.95 & - & $\mathrm{NL}$ & 0.0468 & 1.047 & 0.15 & 0.012 & 0.92 & - & $\mathrm{NL}$ \\
\hline Micropogonias furnieri & 4.5867 & 0.6869 & 5.3525 & 0.261 & 0.73 & - & $\mathrm{NL}$ & 1.9403 & 0.287 & 3.96 & 0.09 & 0.74 & - & $\mathrm{NL}$ \\
\hline Paralonchurus brasiliensis & 0.1699 & 2.4426 & 3.3286 & 0.498 & 0.85 & I & $\mathrm{NL}$ & 0.257 & 1.166 & 0.28 & 0.037 & 0.88 & - & $\mathrm{NL}$ \\
\hline Stellifer naso & 0.2826 & 1.0533 & 0.1632 & 0.023 & 0.8 & - & $\mathrm{NL}$ & 0.1954 & 0.015 & 0.01 & 0.002 & 0.78 & - & L \\
\hline \multirow[t]{3}{*}{ Polydactylus virginicus } & 0.2068 & 1.043 & 0.1971 & 0.0094 & 0.98 & - & $\mathrm{NL}$ & 0.1294 & 1.065 & 0.16 & 0.014 & 0.93 & - & $\mathrm{NL}$ \\
\hline & \multicolumn{7}{|c|}{ BW-WO } & \multicolumn{7}{|c|}{ TL-OL } \\
\hline & $\mathrm{a}$ & SE $a$ & $b$ & SE $b$ & $r^{2}$ & G & Model & $\mathrm{a}$ & SE $a$ & $b$ & SE $b$ & $r^{2}$ & G & Model \\
\hline Pellona harroweri & 15.0327 & 1.9515 & 1364.5 & 227.826 & 0.84 & + & L & -2.9696 & 0.5966 & 0.2196 & 0.0417 & 0.83 & - & L \\
\hline Notarius grandicassis & 13.247 & 1.4257 & 2.8074 & 0.0945 & 0.87 & + & $\mathrm{NL}$ & 0.0044 & 0.0003 & 0.0356 & 0.0086 & 0.97 & - & L \\
\hline Trichiurus lepturus & 0.0008 & 0.0006 & $4 \mathrm{E}-05$ & $5 \mathrm{E}-06$ & 0.89 & I & $\mathrm{NL}$ & -0.0066 & 0.0695 & 0.0076 & 0.0012 & 0.95 & - & L \\
\hline Haemulon plumierii & 426.234 & 1.0712 & 0.5443 & 0.0328 & 0.96 & - & $\mathrm{NL}$ & 12.8221 & 0.4434 & 46.972 & 2.9262 & 0.96 & + & L \\
\hline Haemulon steindachneri & 0.0735 & 0.0055 & 0.0003 & 0.00004 & 0.82 & - & L & -0.2395 & 0.0334 & 0.0196 & 0.0016 & 0.93 & - & L \\
\hline Bairdiella ronchus & $3.00 \mathrm{E}-11$ & 9.2668 & 3.789 & 0.3609 & 0.95 & + & $\mathrm{NL}$ & -0.0397 & 0.0141 & 0.0078 & 0.0005 & 0.98 & - & L \\
\hline Ctenosciaena gracilicirrhus & 0.0049 & 1.073 & 0.7016 & 0.0202 & 0.98 & - & $\mathrm{NL}$ & 0.082 & 0.0162 & 0.0394 & 0.0012 & 0.98 & - & L \\
\hline Cynoscion jamaicensis & 0.0025 & 1.4058 & 0.8477 & 0.0732 & 0.88 & - & $\mathrm{NL}$ & 0.0941 & 0.0595 & 0.0487 & 0.0029 & 0.97 & - & L \\
\hline Cynoscion microlepidotus & 0.016 & 1.3556 & 0.4604 & 0.0647 & 0.82 & - & $\mathrm{NL}$ & 0.0612 & 0.0077 & 0.0051 & 0.0003 & 0.96 & - & L \\
\hline Macrodon ancylodon & 0.0052 & 1.1351 & 0.6595 & 0.0275 & 0.95 & - & $\mathrm{NL}$ & 0.4223 & 0.0288 & 0.0305 & 0.0012 & 0.96 & - & L \\
\hline Menticirrhus americanus & 0.006 & 1.1943 & 0.6593 & 0.046 & 0.94 & - & $\mathrm{NL}$ & 0.0103 & 1.1219 & 0.8692 & 0.0428 & 0.97 & - & $\mathrm{NL}$ \\
\hline Micropogonias furnieri & 0.8819 & 0.1071 & 2.7139 & 0.0096 & 0.82 & - & $\mathrm{NL}$ & 0.0623 & 0.0096 & 0.0055 & 0.0004 & 0.93 & - & L \\
\hline Paralonchurus brasiliensis & 0.0439 & 0.0036 & 0.0004 & 0.00005 & 0.9 & - & L & 0.0148 & 0.0049 & 0.0062 & 0.0003 & 0.96 & - & L \\
\hline Stellifer naso & 0.0089 & 1.0689 & 0.1532 & 0.0295 & 0.76 & - & $\mathrm{NL}$ & 0.1618 & 0.0247 & 0.0242 & 0.0024 & 0.89 & - & L \\
\hline Polydactylus virginicus & 0.0007 & 1.3338 & 0.5665 & 0.0642 & 0.9 & - & $\mathrm{NL}$ & 0.1634 & 0.0237 & 0.0162 & 0.0011 & 0.96 & - & L \\
\hline
\end{tabular}


Table 2. Continued.

\begin{tabular}{|c|c|c|c|c|c|c|c|c|c|c|c|c|c|c|}
\hline \multirow{2}{*}{ Species } & \multicolumn{7}{|c|}{ TL-OW } & \multicolumn{7}{|c|}{ WO-TL } \\
\hline & $\mathrm{a}$ & SE $a$ & $\mathrm{~b}$ & SE $b$ & $r^{2}$ & $G$ & Model & $a$ & SE $a$ & $b$ & $\mathrm{SE} b$ & $r^{2}$ & $G$ & Model \\
\hline Pellona harroweri & -0.2477 & 0.026 & 3.69 & 0.376 & 0.93 & 1 & L & 255.881 & 41.48 & 12.116 & 0.3553 & 0.85 & + & $L$ \\
\hline Notarius grandicassis & 0.0037 & $3.00 \mathrm{E}-04$ & 0.03 & 0.008 & 0.96 & - & L & 2.5465 & 1.1177 & 1.6438 & 0.0296 & 0.9 & - & L \\
\hline Trichiurus lepturus & 0.0492 & 0.0224 & 0.0022 & 0.0004 & 0.94 & - & L & 0.8843 & 1.0286 & 0.0333 & 0.0069 & 0.74 & - & $\mathrm{NL}$ \\
\hline Haemulon plumierii & -3.6941 & 0.221 & 2.1 & 0.17 & 0.93 & - & L & $1.00 \mathrm{E}-08$ & 2.1111 & 5.3811 & 0.2499 & 0.98 & + & $\mathrm{NL}$ \\
\hline Haemulon steindachneri & -0.0856 & 0.016 & 0.01 & $8 \mathrm{E}-04$ & 0.93 & - & L & -0.0284 & 0.0191 & 0.0069 & 0.0009 & 0.83 & - & L \\
\hline Bairdiella ronchus & -0.0797 & 0.0151 & 0.0076 & 0.0005 & 0.97 & - & L & $3.61 \mathrm{E}-17$ & 50.065 & 10.8 & 1.1415 & 0.94 & + & $\mathrm{NL}$ \\
\hline Ctenosciaena gracilicirrhus & 0.1517 & 0.0166 & 0.0262 & 0.0013 & 0.95 & - & L & 0.0001 & 1.3115 & 2.436 & 0.1056 & 0.96 & - & $\mathrm{NL}$ \\
\hline Cynoscion jamaicensis & 0.1065 & 0.019 & 0.02 & $9 \mathrm{E}-04$ & 0.97 & - & L & 0.00002 & 1.8332 & 2.9321 & 0.202 & 0.92 & 1 & $\mathrm{NL}$ \\
\hline Cynoscion microlepidotus & 0.0332 & 0.005 & 0.0026 & 0.0002 & 0.93 & - & L & 0.0009 & 1.7738 & 1.6016 & 0.1835 & 0.87 & - & $\mathrm{NL}$ \\
\hline Macrodon ancylodon & 0.048 & 0.0234 & 0.021 & 0.001 & 0.94 & - & L & 0.0001 & 1.3917 & 2.234 & 0.1044 & 0.94 & - & $\mathrm{NL}$ \\
\hline Menticirrhus americanus & 0.0376 & 0.0032 & 0.0032 & 0.0002 & 0.94 & - & L & 0.0001 & 1.5065 & 2.3625 & 0.1527 & 0.95 & - & $\mathrm{NL}$ \\
\hline Micropogonias furnieri & -0.0915 & 0.0298 & 0.0088 & 0.0013 & 0.92 & - & L & $1 \mathrm{E}-06$ & 2.4738 & 3.7761 & 0.2948 & 0.93 & + & $\mathrm{NL}$ \\
\hline Paralonchurus brasiliensis & -0.002 & 0.0061 & 0.0043 & 0.0003 & 0.97 & - & L & 0.0022 & 2.0483 & 1.1875 & 0.2444 & 0.75 & - & $\mathrm{NL}$ \\
\hline Stellifer naso & 0.0075 & 0.046 & 0.03 & 0.005 & 0.71 & - & L & 0.0034 & 1.2313 & 0.5693 & 0.0898 & 0.76 & - & $\mathrm{NL}$ \\
\hline \multirow[t]{3}{*}{ Polydactylus virginicus } & 0.1197 & 0.0119 & 0.0069 & 0.0006 & 0.95 & - & L & 0.00002 & 2.002 & 1.9361 & 0.2292 & 0.89 & - & $\mathrm{NL}$ \\
\hline & \multicolumn{7}{|c|}{ WO-OL } & \multicolumn{7}{|c|}{ WO-OW } \\
\hline & a & SE $a$ & $b$ & SE $b$ & $r^{2}$ & G & Model & a & SE $a$ & $b$ & $\mathrm{SE} b$ & $r^{2}$ & G & Model \\
\hline Pellona harroweri & -95.2863 & 20.063 & 1.0512 & 0.1719 & 0.76 & - & L & 0.6918 & 0.104 & 64 & 12.17 & 0.8 & + & L \\
\hline Notarius grandicassis & 677.397 & 1.9976 & 1.7663 & 0.2428 & 0.92 & - & $\mathrm{NL}$ & 459.89 & 2.281 & 1.88 & 0.323 & 0.87 & - & $\mathrm{NL}$ \\
\hline Trichiurus lepturus & 0.0444 & 1.3164 & 2.6697 & 0.3361 & 0.89 & 1 & $\mathrm{NL}$ & 2.4086 & 2.378 & 3.57 & 0.502 & 0.86 & 1 & $\mathrm{NL}$ \\
\hline Haemulon plumierii & 3.3557 & 1.2519 & 1.7313 & 0.1179 & 0.95 & - & $\mathrm{NL}$ & 25.986 & 1.574 & 2.4 & 0.204 & 0.92 & - & $\mathrm{NL}$ \\
\hline Haemulon steindachneri & 0.0565 & 0.0063 & 0.3489 & 0.0391 & 0.88 & - & L & 0.0365 & 0.01 & 0.71 & 0.094 & 0.84 & - & L \\
\hline Bairdiella ronchus & 617102 & 6.4754 & 8.7848 & 1.1499 & 0.91 & + & $\mathrm{NL}$ & 187418 & 2.238 & 6.92 & 0.425 & 0.98 & + & $\mathrm{NL}$ \\
\hline Ctenosciaena gracilicirrhus & 0.2456 & 1.0538 & 2.8943 & 0.0976 & 0.97 & 1 & $\mathrm{NL}$ & 0.6417 & 1.137 & 3.49 & 0.18 & 0.94 & + & $\mathrm{NL}$ \\
\hline Cynoscion jamaicensis & 0.1006 & 1.0254 & 3.4241 & 0.2167 & 0.93 & + & $\mathrm{NL}$ & 1.1632 & 1.167 & 3.6 & 0.249 & 0.92 & + & $\mathrm{NL}$ \\
\hline Cynoscion microlepidotus & 7.6983 & 1.3784 & 2.3376 & 0.1851 & 0.94 & - & $\mathrm{NL}$ & 34.937 & 2.156 & 2.33 & 0.322 & 0.83 & - & $\mathrm{NL}$ \\
\hline Macrodon ancylodon & 0.0675 & 1.0254 & 3.3869 & 0.1557 & 0.94 & + & $\mathrm{NL}$ & 0.4269 & 1.105 & 2.28 & 0.162 & 0.87 & - & $\mathrm{NL}$ \\
\hline Menticirrhus americanus & 33.7587 & 1.2794 & 2.7191 & 0.1097 & 0.98 & - & $\mathrm{NL}$ & 1604.8 & 2.6 & 4.03 & 0.386 & 0.89 & + & $\mathrm{NL}$ \\
\hline Micropogonias furnieri & 727.085 & 2.1282 & 5.0159 & 0.441 & 0.92 & + & $\mathrm{NL}$ & 23.157 & 1.476 & 2.15 & 0.162 & 0.94 & - & $\mathrm{NL}$ \\
\hline Paralonchurus brasiliensis & -0.5903 & 0.0218 & 0.1707 & 0.1293 & 0.75 & - & L & 0.003 & 1.81 & 1.24 & 0.234 & 0.78 & - & $\mathrm{NL}$ \\
\hline Stellifer naso & -1.5281 & 0.039 & 0.953 & 0.1002 & 0.87 & - & L & 0.0057 & $6.00 \mathrm{E}-04$ & 0.03 & 0.002 & 0.92 & - & L \\
\hline Polydactylus virginicus & 0.0616 & 1.2884 & 2.8049 & 0.3601 & 0.87 & I & $\mathrm{NL}$ & 0.7464 & 1.957 & 3.33 & 0.501 & 0.83 & I & $\mathrm{NL}$ \\
\hline
\end{tabular}

BW: Body weight, TL: Total length, OL: otolith length, OW: otolith width, WO: weight of the otolith, SE: standard error, G: growth type = -: negative allometric, +: positive allometric, I: isometric, NL: Nonlinear equation, L: linear equation. $b$ values in bold are significant $(t-t e s t ; p<0.05)$.

despite the strong biometric relationships derived from our data, our estimated parameters should be used with caution, as our small sample sizes (mainly those $\leq 10$ for $B$. ronchus, P. harroweri, N. grandicassis, Trichiurus lepturus, and Paralonchurus brasiliensis) and a selective effect of the mesh size used by the shrimp trawlers may have caused the size distributions in our samples to be underrepresented.

The lack of statistical differences between left and right sagitta indicates that otoliths on either body side are indistictively usable for fish-size estimations (Battaglia et al. 2010; Mehanna et al. 2016; Park et al. 2018; Yilmaz et al. 2015). The high coefficients of determination for the relationships between otolith measurements and fish size in all our species indicates that length or weight of fish can be reliably estimated from otoliths found in stomach contents of predators. Our values of $b$ varied considerably among the species, owing to the variable size and shape of the sagittae among the species. Yet, at family level (e.g. Scianidae), the $b$ values tended to negative allometry in most relationships, as species within families are relatively more similar in shape. Most studies providing relationships between otolith and fish size have used only the width and length of the otolith (Giménez et al. 2016; Assis et al. 2018; Park et al. 2018). Considering the high values of correlation in our analyses, the inclusion of otolith weight in our estimations contributed to strengthen the relationship models.

The relationship between otoliths and fish size was estimated for species from different regions around the 
world, such as Mexico (De La Cruz-Agüero et al. 2016), India (Aneesh Kumar et al. 2017), Turkey (Altin and Ayyildiz 2017), Montenegro (Kanjuh et al. 2018) and Australia (Park et al. 2018). However, studies on this subject are still incipient for the South Atlantic. An analysis of the otolith-fish size relationship for juveniles of four species of Scianidae in the De la Plata River estuary, in southern Uruguay (Waessle $e t$ al. 2003), included three of our species (M. ancylodon, M. furnieri, and $P$. brasiliensis), which showed similar parameter values to those estimated in our study. Assis et al. (2018) analyzed seven species of commercial importance from the coast of Bahia, in northeastern Brazil, and Souza et al. (2019) studied Pomatomus saltatrix from the coast of Rio de Janeiro in the southeast. Besides their economic importance, some of these fish species, such as $M$. ancylodon, $M$. americanus, $M$. furnieri, $P$. harrowerri, and T. lepturus, are common prey of the estuarine dolphin, Sotalia guianensis (Van Bénéden, 1864), that occurs along the Brazilian coast (Vieira 2014), further highlighting the importance of our biometric estimators for ecological studies.

\section{CONCLUSIONS}

This study is a contribution to the knowledge about the relationships between otolith and fish size in 15 fish species from the northern Brazilian coast for the reliable estimation of species-specific fish length or weight from otolith size. Our results form a baseline for future studies on trophic ecology and fish distribution, and will enable a more accurate evaluation of length and/or biomass of demersal fishes consumed by predators.

\section{ACKNOWLEDGMENTS}

This study was funded by the CEPNOR-ICMBio and Conselho Nacional de Desenvolvimento Científico e Tecnológico (CNPq) (n 71/2013). Thanks to M. Petrere Jr. for their valuable comments to improve the manuscript, and Carson Jeffres (University of California, Davis) for English revision of the final version of the manuscript. RRSO is funded by Conselho Nacional de Desenvolvimento Científico e Tecnológico (CNPq). FSM is funded by Coordenação de Aperfeiçoamento de Pessoal de Nível Superior (CAPES). MCA is funded by a PNPD postdoctoral fellowship at the Graduate Program in Aquatic Ecology and Fisheries (PPGEAP) of Universidade Federal do Pará - UFPA (PNPD/CAPES \# 06/2017) and acknowledges support from the Biodiversity Conservation Organization IDEA WILD. TG receives a productivity grant from $\mathrm{CNPq}$ (310299/2016-0).

\section{REFERENCES}

Altin, A.; Ayyildiz, H. 2017. Relationships between total length and otolith measurements for 36 fish species from Gökçeada Island, Turkey. Journal of Applied Ichthyology, 34: 136-141.
Aneesh Kumar, K.V.; Deepa, K.P.; Hashim, M.; Vasu, C.; Sudhakar, M. 2017. Relationships between fish size and otolith size of four bathydemersal fish species from the south eastern Arabian Sea, India. Journal of Applied Ichthyology, 33: 102-107.

Assis, D.A.S.; Santos, J.A.; Moraes, L.E.; Santos, A.C.A. 2018. Biometric relation between body size and otolith size of seven commercial fish species of the south-western Atlantic. Journal of Applied Ichthyology, 34: 1176-1179.

Barletta, M.; Jaureguizar, A.J.; Baigun, C.; Fontoura, N.F.; Agostinho, A.A.; Almeida-Val, V.M.F.; et al. 2010. Fish and aquatic habitat conservation in South America: a continental overview with emphasis on neotropical systems. Journal of Fish Biology, 76: 2118-76.

Battaglia, P.; Malara, D.; Romeo, T.; Andaloro, F. 2010. Relationships between otolith size and fish size in some mesopelagic and bathypelagic species from the Mediterranean Sea (Strait of Messina, Italy). Scientia Marina, 74: 605-612.

Campana, S.E. 2004. Photographic atlas of fish otoliths of the Northwest Atlantic Ocean. NRC Research Press, Ottawa, 284p.

De La Cruz-Agüero, J.; Chollet-Villalpando, J.G.; Valle-López, F.L. 2016. Relationships between sagittal otolith length and fish size for 14 Mojarra species (Gerreidae: Perciformes) in Mexico. Turkish Journal of Fisheries and Aquatic Sciences, 16: 651-657.

De Pierrepont, J.F.; Dubois, B.; Desormonts, S.; Santos, M.B.; Robin, J.P. 2005. Stomach contents of English Channel cetaceans stranded on the coast of Normandy. Journal of the Marine Biological Association of the United Kingdom, 85: 1539-1546.

Froese, R. 2006. Cube law, condition factor and weight-length relationships: history, meta-analysis and recommendations. Journal of Applied Ichthyology, 22: 241-253.

Froese, R.; Tsikliras, A.C.; Stergiou, K.I. 2011. Editorial note on weight-length relations of fishes. Acta Ichthyologica et Piscatoria, 41: 261-263.

Giménez, J.; Manjabacas, A.; Tuset, V.M; Lombarte, A. 2016. Relationships between otolith and fish size from Mediterranean and north-eastern Atlantic species to be used in predator-prey studies. Journal of Fish Biology, 89: 2195-2202.

Giarrizzo, T.; Krumme, U. 2008. Heterogeneity in intertidal fish fauna assemblages along the world's longest mangrove area in northern Brazil. Journal of Fish Biology, 72: 773-779.

Kanjuh, T.; Mrdak, D.; Piria, M.; Tomljanović, T.; Joksimović, A.; Talevski, T.; Milošević, D. 2018. Relationships of otolith dimension with body length of European eel Anguilla anguilla (Linnaeus, 1758) from Adriatic catchment of Montenegro. Acta Adriaica, 59: 91-96.

Harvey, J.T.; Loughlin, T.R.; Perez, M.C.; Oxman, D.S. 2000. Relationship between fish size and otolith length for 63 species of fishes from the Eastern North Pacific Ocean. NOAA Technical Report nr. 150, NOAA/National Marine Fisheries Service, Seattle, 36p.

Isaac, V.J.; Braga, T.M.P. 1999. Rejeição de pescado nas pescarias da Região Norte do Brasil. Arquivos de Ciências do Mar 32: 39-54.

Lombarte, A.; Chic, O.; Parisi-Baradad, V.; Olivella, R.; Piera, J.; Garcia-Ladona, E. 2006. A web-based environment for shape 
analysis of fish otoliths. The AFORO database. Scientia Marina 70: $147-152$.

Marceniuk, A.P.; Rotundo, M.M.; Caires, R.A.; Cordeiro, A.P.B.; Wosiacki, W.B.; Oliveira, C.; et al. 2019. The bony fishes (Teleostei) caught by industrial trawlers off the Brazilian North coast, with insights into its conservation. Neotropical Ichthyology, 17: $1-28$.

Mehanna, S.F.; Jawad, L.A.; Ahmed, Y.A.; Abu El-Regal, M.A.; Dawood, D. 2016. Relationships between fish size and otolith measurements for Chlorurus sordidus (Forsskål, 1775) and Hipposcarus harid (Forsskål, 1775) from the Red Sea coast of Egypt. Journal of Applied Ichthyology, 32: 356-358.

Park, J.M.; Gaston, T.F.; Riedel, R.; Williamson, J.E. 2018. Biometric relationships between body and otolith measurements in nine demersal fishes from north-eastern Tasmanian waters, Australia. Journal of Applied Ichthyology, 34: 801-805.

Popper, A.N.; Ramcharitar, J.; Campana, S.E. 2005. Why otoliths? Insights from inner ear physiology and fisheries biology. Marine and Freshwater Research, 56: 497-504.

Souza, G.M.; Tubino, R.A.; Monteiro-Neto, C.; Costa, M.R. 2019. Relationships between fish and otolith dimensions of Pomatomus saltatrix (Linnaeus, 1766) (Perciformes: Pomatomidae) in southeastern Brazil. Neotropical Ichthyology, 17: e180032.
Tuset, V.M.; Piretti, S.; Lombarte, A.; González, J.A. 2010. Using sagittal otoliths and eye diameter for ecological characterization of deep-sea fish: Aphanopus carbo and A. intermedius from NE Atlantic waters. Scientia Marina 74: 807-814.

Vieira, J.O. 2014. Diferenças alimentares em populações de Boto-cinza Sotalia guianensis (Van Benédén, 1864) (Cetacea, Delphinidae) nas Costas Norte e Nordeste Brasileira. Master's dissertation, Universidade Federal do Pará and Museu Paraense Emílio Goeldi, Brazil, 49p.

Waessle, J.A.; Lasta, C.A.; Favero, M. 2003. Otolith morphology and body size relationships for juvenile Sciaenidae in the Río de la Plata estuary (35-36 S). Scientia Marina, 67: 233-240.

Yilmaz, S.; Yazicioğlu, O.; Yazici, R.; Polat, N. 2015. Relationships between fish length and otolith size for five cyprinid species from Lake Ladik, Samsun, Turkey. Turkish Journal of Zoology, 39: 438-446.

RECEIVED: $26 / 02 / 2019$

ACCEPTED: 09/08/2019

ASSOCIATE EDITOR: Helder M. Espírito Santo 